\title{
The Design of Disease Monitoring System for Chinese Outbound Tourism: A Discussion from Public Management Perspective
}

\author{
Jing-Jing GUAN ${ }^{1, a}$, Ming XIA ${ }^{2, b,{ }^{*},}$ Julian-K. AYEH ${ }^{3, c}$ \\ ${ }^{1}$ School of Tourism \& City Management, Zhejiang Gongshang University, 18th Xuezheng Street, \\ Hangzhou, China \\ ${ }^{2}$ Faculty of Pharmacy, Zhejiang Chinese Medical University, 84\# mailbox Binwen Road, Hangzhou, \\ China \\ ${ }^{3}$ Department of Hospitality and Tourism Management, University of Cape Coast, Cape Coast, \\ Ghana. \\ ajjguan66@126.com, bxia1ming2@126.com, ${ }^{c} j k$ kayeh@gmail.com \\ ${ }^{*}$ Corresponding author
}

Keywords: Chinese outbound tourism, communicable disease, disease monitoring system

\begin{abstract}
Over the past decade, China has been one of the fastest-growing tourism source markets in the world. The volume of Chinese outbound travelers has grown from 10 million in 2000 to 83 million in 2012. However, the growth of outbound tourists increases the likelihood of the spread of communicable diseases which significantly influence tourism industry. The evidences can be seen from the Foot-Mouth Disease in UK and SARS in China. This paper proposes a framework of disease monitoring system for outbound tourism by analyzing the direction of tourists flow, the transmission of information flow, and the connection between information flow and tourists flow. In addition, the necessary factors that ensure a successful operation of the disease monitoring system are discussed.
\end{abstract}

\section{Introduction}

The Tourism sector has been one of the most important economic sectors around the world. In 2012, international tourism receipts hit a new record reaching an estimated US\$ 1,075 billion worldwide, up 4\% in real terms, from US\$ 1,042 billion in 2011. The year 2012 was also the first time that international tourist arrivals exceeded 1 billion, which is 4\% increase from 2011[1]. Globally, as an export sector, tourism ranks fourth after fuels, chemicals and food. Tourism exports account for as much as $30 \%$ of the world's exports of commercial services and $6 \%$ of overall exports of goods and services. Over the last decade, China has been one of the fastest-growing tourism source markets in the world. The volume of international trips by Chinese travelers has grown from 10 million in 2000 to 83 million in 2012. Overseas expenditure by Chinese tourists who have become the largest spenders in international tourism globally has increased almost eightfold since 2000[2].

The booming tourism industry can be significantly influenced by communicable diseases. For example, the foot-mouth disease outbreak in UK, 2001 was one of the largest crises that the UK tourism industry endured for many years. At the height of the disease, the estimated losses of revenue for the UK tourism industry were to be running at 179 million pounds in a week, or $13 \%$ of normal weekly tourism expenditures [3]. Figures released from the International Passenger Survey showed that overseas visitor numbers for UK were lower in the first 6 months of 2001 compared with 2000. British hoteliers, as well as airline carriers, reported a 10 percent decline in reservations from inbound tourists [4]. Similarly, in 2003, the outbreak of Severe Acute Respiratory Syndromes (SARS) in China significantly influenced Chinese tourism industry. From March to May, 2003, the declines ofoverseas' tourist arrivals in China were 7.2, 30.0 and 31.0 percentages respectively, 
compared to the same months of 2002. In April and May, the amounts of overnight foreign tourists fell by 53.52 percent and 70.50 percent from the year-earlier period. From June afterward, even when the SARS was effectively controlled, overseas' tourist arrivals still had negative growths compared with the same periods of the previous year. The tourism industry of China did not recover until December, 2003[5]. Recently, the outbreak of avian influenza in southeast China also caused the cancellations of group tours to southeast destinations, and the exact losses of tourism industry remain unknown.

The movements of tourists are one of the important channels of disease transmission, especially communicable diseases. For instant, the dengue had not been observed since 1940s in China, while in 1978, the outbreak of W dengue in Foshan, Guangdong province, was confirmed to be imported from a foreign country by a traveler[5]. According to reports from the Office of Frontier Health and Quarantine (OFHQ), tourists are the third largest group carrying communicable disease (after staff on international vehicles and overseas workers) [6]. Therefore, for the consideration of tourists' health, the negative influence on tourism industry and the spread of communicable diseases, outbound travelers have to be monitored in respect of diseases they may be carrying.

This paper, by analyzing the direction of outbound tourists flow, the connection between information flow and tourists flow, as well as the medium between information flow and tourists flow, proposes a framework of disease monitoring system for outbound tourism. The paper was structured as follows. The next section discusses the problems of present disease monitoring systems for outbound tourism in China. A framework of disease monitoring system for outbound tourism is proposed in the third section with elaboration on the mechanism of the system. Issues influencing the successful operation of the disease monitoring system are then discussed in the last section with a brief conclusion.

\section{A Review of Current Disease Monitoring System for Chinese Outbound Tourism}

Two organizations, namely the office of frontier health and quarantine (OFHQ) and the international healthcare center (IHC), are in charge of the control of communicable diseases and the monitoring and prevention of international disease epidemic crossing national boundaries. The OFHQ which is normally situated at the national entry/exit port, such as international airport, dock, and train terminal, is mainly responsible for the detection and quarantine of international travelers' health situation. According to the law of frontier health quarantine of the People's Republic of China, which was promulgated in 1989 and offers a legal ground for the disease monitoring of international travelers, the establishment of OFHQ at every international entry/exit port is compulsory.

The major responsibility of IHC is to vaccinate outbound travelers who apply for a vaccination. In other words, the vaccinations for outbound tourists are not compulsory, except for tourists visiting destinations that are regarded as highly risky in respect of disease infections. The IHCs are mainly located in the major cities of China, therefore travelers from small cities or rural areas are inconvenienced in receiving the service from IHCs. The OFHQ and IHC are two cornerstones which form the present monitoring system for travel disease of China. Due to the increase of Chinese outbound tourists, the present monitoring system shows some limitations.

\section{Being Informed rather than Inspecting health Conditions.}

Both the OFHQ and the IHC have worked passively in the current monitoring system. The OFHQ asks tourists to report their health conditions and infection histories by filling in the "health declaration card". Yet, most tourists do not realize the importance of disease report and lack the motive to offer self-report, since the self-report of disease might lead to problems such as being 
quarantined or being monitored. Therefore, self-report of disease infection is not very reliable in terms of the discovery of infectious victims and vectors. Limited tourists would actively apply for vaccination, since the vaccination is optional. In most cases, tourists only have the intentions of vaccination in two situations. First, the countries where tourists intend to visit are on the alert of communicable disease. Second, the authorities from tourists' destinations have such a requirement. Without vaccination, the visas may not be issued. In conclusion, both OFHQ and IHC are passively informed by tourists rather than actively inspecting health conditions. As the outbound tourists may not actively apply for vaccination before their trips, and also may not actively report their conditions of disease-carrying, OFHQ and IHC cannot fully obtain the knowledge of tourists' health situations and the potential risks of the disease transmission.

\section{Insufficient Usage of Advanced Test Technique of Communicable Disease.}

Currently, rapid temperature detection and medical inspectors' observations are two major approaches that OFHQ rely on to detect tourists' health conditions. However, these two approaches have their own limitations. Most infected tourists do not have symptoms of fever, when they arrive at the entry ports. Thus the temperature detection is not able to identify the potential patients. The medical inspectors' observations and inquiries are also insufficient in the identification of infected tourists. The limited medical inspectors are not able to inquire on every single tourist, since the volume of tourists could be thousands and millions. Also the effectiveness of observation and inquiry is lean on the expertise of medical inspectors which are varied and unstable. Although there are great advances in the test techniques of communicable diseases, for example, the developments of Enzyme linked immunosorbent assay (ELIA), Nucleid acid sequence-based amplification (NASBA), Loop-mediated Isothermal Amplification (LAMP), and Gene chip, these advanced test techniques cannot be fully applied, due to the high cost, limited laboratories, instruments and technicians.

\section{Incapable of Tracing Tourists in Their Entire Journey.}

The IHC mainly focuses on pre-travel period and asks tourists to vaccinate before their trips. The OFHQ detects tourists who complete their journey and come back to China. In the outbound tourism, when Chinese tourists are visiting foreign countries, both the IHC and OFHQ lose their powers in respect of disease monitoring of tourists. Once tourists were infected or exposed to the risks of communicable diseases at their foreign destinations, if tourists do not actively inform, both IHC and OFHQ are not able to detect. Even when tourists report their illnesses at the entry ports, it might be too late, as passengers and crews on the same vehicle as the infected tourists have the risks of infections. Also, OFHQ may not have sufficient time to response to the emergencies, if the cases are reported on arrival.

\section{Disconnection among Related Organizations.}

In China, the OFHQ and IHC are supervised by the administration of quality supervision inspection and quarantine (AQSIQ), while to monitor the communicable diseases, several organizations need to be involved, such as health administration, bureau of exit and entry administration, tourism administration, and tourism related organizations. However, in the current practice, related organizations do not have good collaboration. For example, when Chinese tourists apply for passports, the bureaus of exit and entry administration do not examine tourists' health certificates. If the health certification is one of the essential documents for passport application, tourists would then be motivated to have physical examinations and vaccinations at the IHCs. Similarly, the tourism administration does not require travel agencies to report tourists' health conditions, and scarcely offers educational programs of disease knowledge to travel agencies and tour guides. Actually, the travel agency, as the professional organization to organize the group tour 
and offer travel service, can play a more important role in the monitoring of diseases.

\section{The Framework of The Disease Monitoring System for Chinese Outbound Tourism}

Fig. 1 presents the framework of disease monitoring system for outbound tourism which was proposed based on the analysis of the outbound tourists flow, the information flow and the media connecting the two flows.

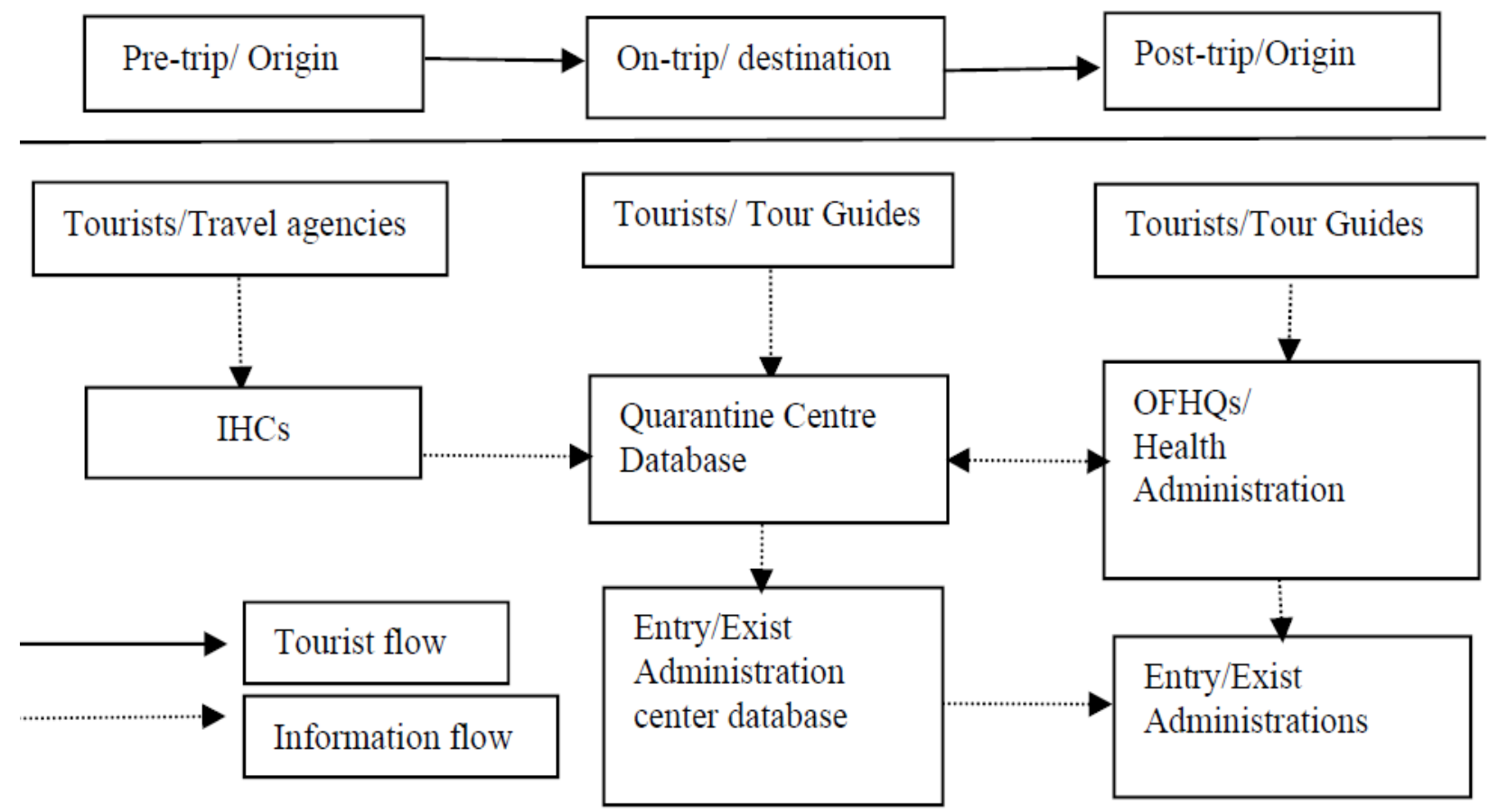

Fig 1 The framework of disease monitoring system for Chinese outbound tourism

\section{Outbound Tourists Flow.}

Tourism is essentially a mobile phenomenon. Tourists leave their original places for tourism destinations which generate tourists flow. In the outbound tourism, the movement of tourists flow was from China to foreign countries, and then back to China. According to the movement of China outbound tourist flow, an entire trip of a tourist can be divided into three phases, namely pre-trip, on-trip and post-trip (See Fig 1). As stated above, OFHQ mainly focuses on the phase of post-trip and IHC is responsible for the phase of pre-trip. For the phase of on-trip, it is extremely difficult for two Chinese administrations to actively inspect outbound Chinese tourists. Hence, one objective of the disease monitoring system is to assist related administrations to trace the health conditions of outbound tourists.

\section{Information Flow.}

The information flow refers to the movement of information about tourists' health conditions. In different phases of travel, the receiver of the information is different. On the phase of pre-trip, the information on tourists' health conditions goes to the IHC. However, due to the disconnection of database between different departments, the IHC would not share the information with the OFHQ and entry/exit administration. The only way that OFHQ and the entry/exit administration have the information of tourists' health conditions from the IHC is by health certifications brought by tourists.

In the on-trip phase, tourists are in foreign countries, therefore the most effective way would be to use the Internet to collect the information on tourists' health conditions. However, there is no exiting information system opening to the public. One information system that is used in China to 
report the detection of communicable diseases hierarchically is the information management system of health quarantine at entry/exit port established in 2007. It is a system that is only opened to the OFHQ. In other words, tourists do not have the authorizations to report their conditions of infectious disease via the information system.

At the post-trip stage, the information of tourists'health conditions is mainly known by the OFHQ. Once the communicable disease has been detected, the OFHQ must use the information management system of health quarantine at entry/exit port to report the epidemic to its superior (i.e local AQSIQ). The information is then reported hierarchically to the general AQSIQ. Meanwhile, the health administration has its own information system (i.e. the disease monitoring system) for the monitoring of the communicable diseases around the nation, which is only opened to the hospitals and health administration. Since these two systems are disconnected, the health administration do not realize the outbreak of communicable diseases until the infected tourists are sent to the hospitals, and hospitals report diseases via the monitoring system.

\section{The Informants of the Information.}

Due to the large numbers of outbound tourists, it is extremely difficult for the OFHQ or IHC to detect the heath conditions of each tourist. Therefore, no matter which phase of the trip, tourists are the main informants in reporting the information of the disease infection. As stated above, for some concerns, tourists may not have the motive to actively report disease infection. To address the problem, travel agencies and tour guides need to be involved in the process of disease reporting. Currently, most Chinese tourists still travel overseas with group tours organized by travel agencies. Hence, travel agencies can be an important source to report tourists' disease infections to.

\section{Functionalization of the System.}

The key of the disease monitoring system is a central database that is shared by different organizations involved in the tourists disease monitoring. Each organization is assigned a specific ID to login the database. In the database, the information of tourists can be traced based on the passport ID of each tourist.

In the pre-trip phase, travel agencies need to organize tourists who intend to visit countries with high risks of epidemic to have vaccinations. The IHC uses the passport IDs of tourists as the keyword to document the records of vaccinations in the central database. During the journey, if tourists had or have been exposed to some infectious diseases, such as cholera, dysentery, and malaria, the tour guides are required to report the situation via the central database using the IDs of travel agencies. The tour guides also need to fill in the necessary information, such as tourists $>$ passport IDs, the schedules of group tours, etc. Once the database receive the information, administrators of the database need to evaluate reports and warn the OFHQ, AQSIQ, health administration and entry/exit administration of the destinations where the transport carrying the infected tourists will be abroad. When tourists return to China, the OFHQ can particularly focus on the transport vehicles and infected tourists in the database and the tourists who are on the same vehicle as infected. The central database also links to the system of entry/exit administration and exchanges the information. Once the officers of the entry/exit administrations input the passport IDs into the system, if the tourists are risky in terms of carrying communicable diseases, the system can reminder the officers to pay attention on these tourists and inform officers of the OFHQ for further examinations. At the same time, the local health administration also has been altered via the central database and can inform the local hospital for preparation.

\section{Discussion \& Conclusion}

The paper proposed a framework of the disease monitoring system for outbound tourism. The 
construction of the central database can collect the information of tourists' health conditions from pre-trip, on-trip and post-trip stages, and connect various organizations. The successful running of the system does not only rely on the construction of physic facility, but also requires other factors, including the health education for tourists, the improvement of law and the redesign of administration systems.

\section{Tourists Education.}

Without effective approach actively monitoring tourists, the disease monitoring system still need to collect the information based on tourists or tour guides' active reports. Therefore, tourists and tour guides must be educated to realize the importance of disease reports, have the correct attitude towards vaccination and disease infection report, and behave appropriately in emergency situations. The education programs could include the knowledge of communicable disease symptoms, the major communicable diseases in the destinations, how to report the disease infections, how to handle infected tourists or vectors, and the way of cutting the routes of disease transmission.

\section{The Improvement of the Law.}

Currently, China has one law and several regulations related to communicable diseases. Besides, regulations are specific about certain diseases, such as HIV and SARS. Some law and regulations are more general, such as the Law of the People's Republic of China On the Prevention and Control of Infectious Diseases, Measures for The People's Republic of China On the Prevention and Control of Infectious Diseases, and Regulation on the Urgent Handling of Public Health Emergencies. These law and regulations mainly describe the responsibilities of public organizations and do not regulate the responsibilities of individuals and private organizations. Tourists, tour guides or travel agencies would not be punished, even when they conceal the emergence of diseases. Hence, the regulations need to give more clear descriptions on individuals and private organizations' responsibilities. For those having intentional concealments, punishments could be applied accordingly.

\section{The Cooperation of Different Organizations.}

One factor that leads to the ineffectiveness of the current administration system is the insufficient cooperation between different organizations. For example, the OFHQ \& IHC are supervised by the AQSIQ, the entry/exit administration who are able to examine every tourist is supervised by the administration of public security, the further treatments of diseases are responsibilities of hospitals which are supervised by the health administrations, and the outbound tourism is administrated by tourism bureaus. Therefore, these administrations need to have a tighter cooperation, for example, building the central database cooperatively to exchange information, forming the regulation jointly that defines the responsibilities of different public administrations, traveler and travel related organizations such as travel agency, transportation corporation, and educating the public collaboratively.

\section{Conclusion}

The boosting of China's outbound tourism generates millions of tourists, as well as great risk for the communicable disease transmission. To monitor the transmission of diseases, it is critical to exchange information among different administrations, and among tourists and administrations. A disease monitoring system with a central database to transfer the information and to involve key organizations will make the monitoring of communicable diseases with outbound tourism more efficient. The education programs, the improvement of law and the cooperation of different organizations also will facilitate the efficiency of disease detection. Furthermore, with the innovation of the quicker quarantine techniques, in the future every tourist might be able to have a 
quick examination at the entry port. At that time, most communicable disease can really be detected and prevented.

\section{Acknowledgment}

This research is financially supported by Foundation of China Tourism Bureau (No.13TACG010).

\section{References}

[1] UNWTO, World Tourism Barometer, 2013, 1-3.

[2] UNWTO, China - the new number one tourism source market in the world, Apr 2013.

[3] Irvine, W., Anderson, A., The impacts of Foot and Mouth Disease on a peripheral tourism area: The role and effect of crisis management, Journal of Travel and Tourism Marketing, 19(2005): 47 60 .

[4] Baxter, E., Bowen, D., Anatomy of tourism crisis: explaining the effects on tourism of the UK Foot and Mouth Disease epidemics of 1967-68 and 2001 with special reference to media portrayal, International Journal of Tourism Research, 6(2004): 263-273.

[5] Wan, HL., The relationship between tourism and infectious diseases \& prophylaxis and treatment, Master thesis, Normal University of Shanxi. (In Chinese)

[6] Wang, HH., et al., Analysis on disease surveillance results among international travelers at Wuhan ports, Chinese Frontier Health Quarantine, 6(2011): 439-442. (In Chinese) 